\title{
Comparison on mechanical properties of lignocellulosic flour epoxy composites prepared by using coconut shell, rice husk and teakwood as fillers
}

\author{
S. Sajith ${ }^{\mathrm{a}}$, V. Arumugam ${ }^{\mathrm{a}}$, H. N. Dhakal ${ }^{\mathrm{b} *}$

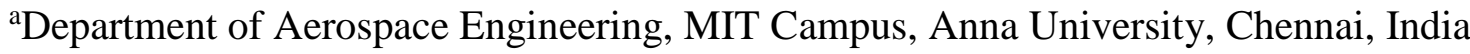 \\ ${ }^{\mathrm{b}}$ Advanced Polymer and Composites (APC) Research Group, School of Engineering, \\ University of Portsmouth, PO1, 3DJ, UK.
}

\begin{abstract}
The objective of this work was to investigate the feasibility of using lignocellulosic fillers (coconut shell, rice husk and teakwood) as alternative reinforcement for expensive nonrenewable fillers being used in conventional composites. Various concentrations of epoxy based bio-fillers composites were prepared and the influences of filler size (75-105 and 106$180 \mu \mathrm{m})$ and mass concentrations (2.5 and $4.5 \mathrm{wt} . \%)$ on the mechanical properties were investigated. Properties such as deformability, stiffness, elasticity and strain energy absorption ability of each material combination were determined using single indentation load control, single indentation displacement control and multi-cyclic indentation tests. The results showed that parameters such as filler size, volume content, filler type (chemical composition and shape) dispersion influenced these properties. It was revealed that high lignin content increased the stiffness whereas high cellulose content with high impregnation ability favoured deformability. High cellulose content and surface roughness supported better adhesion due to a large number of hydrogen bonds and high mechanical interlocking respectively.
\end{abstract}

Keywords: A. Particle-reinforced composites, B. Adhesion, C. Deformation, D. Hardness testing

\footnotetext{
* Corresponding author. Tel: + 44 (0) 239284 2582; fax: + 44 (0) 2392842351.

E-mail: hom.dhakal@port.ac.uk
} 


\section{Introduction}

Fibre reinforced plastic (FRP) composite materials are increasingly replacing the conventional metallic materials due to their unique attributes over conventional metals in terms of high stiffness to weight ratio, andtailorability to meet the exact needs of the structures. Due to these attractive attributes, composite materials have gained important applications in aerospace, automotive and marine sectors. Despite of having these numerous advantages, laminated composite structures are susceptible to fracture, commonly called delamination. Delamination is always associated with the initiation and propagation of cracks in the interlaminar space which is occupied by matrix material. There are many reported works on techniques to arrest or delay delamination, which include using hybrid systems by using different reinforcements [1], the addition of expensive carbon nanotubes into the matrix material to toughen and deviate the crack [2]. Toughening involves improving the elastic strength as well as ductility in an attempt to have high energy absorbing capability. Investigations have been carried out to find a cheap alternative to carbon nanotubes but, at the same time, also have synergistically good strength, toughness, shear strength and other mechanical properties [3, 4].

Furthermore, investigation on inclusion of commercial fillers like carbon nanotube, carbon black (CB), thermally reduced graphite oxide (TRGO), alumina and $\mathrm{TiO}_{2}$ nanoparticles polymer matrix have been carried out $[5,6]$. The reported works suggest that optimum content of filler improved the mechanical properties such as tensile, flexural and shear strength as well as modulus of the matrix. Properties influenced by material surface like fracture resistance, abrasion, micro and macro hardness were also reported to be increased. Carbon black enabled polymer chain sliding which in turn influenced deformability of matrix, but when added in higher concentrations the properties deteriorated due to agglomeration of fillers, higher viscosity and difficulty in dispersion [7, 8]. 
In recent years, due to ecological concerns, bio-fillers, which are cost effective, provide ecofriendly alternatives to non-renewable petroleum reinforcements and, in many cases, are considered as waste, are being used to modify polymer matrices to acquire improved mechanical properties such as stiffness, elastic modulus, toughness and fracture resistance. Certain bio-filler based particulate composites have shown improvement in flexural strength, flexural modulus and thermal stability. The work carried out by Fombuena et al. [9] on seashell waste and Huang et al. [10] on sugarcane based biocomposites suggests that inclusion of seashell and rice husk bio-fillers to epoxy improved hardness, tensile modulus, impact energy, erosion resistance and thermal properties. Remarkable increase in yield stress, tensile strength, toughness and thermal degradation temperature were reported for lignin based date palm wood powder and rice husk filled glass epoxy particulate polymer composites $[11,12]$. Cellulose in bio-fillers improves mechanical properties whereas lignin, which is rigid, reduced water absorption and improved thermal stability. Also, rod shaped fillers exhibited higher tensile modulus and strength than the elliptical shaped fillers [13-16]. Chemical compositions of various lingnocellulosic fillers have been determined and standard methods for filler preparation and quasi-static indentation and have been discussed $[17,18]$.

In addition, experimental investigations on certain biofillers such as sugarcane bagasse, rice husk, coconut coir, rice straw and egg shell, and the influence of filler content, size, shape and chemical composition on prevalent mechanical properties like tensile, flexural, impact and fracture toughness have been carried out [19-24]. Notwithstanding, there is still a large gap in investigating lignocellulosic fillers resources, their influential parameters and effects on the properties which could possibly be very useful for material evolution. In order to address this gap, this study focuses on three different types of lingo-cellulosic fillers: rice husk, coconut shell and teakwood. They were subjected to indentation testing to study the influence of filler mass percentage, size, shape, surface properties and chemical 
composition on the important mechanical properties such as elasticity, deformability, stiffness, strain energy absorption and restoring capabilities. The results obtained will provide knowledge about what kind of filler material and filler content will be best suited for applications that require certain tailored material properties like stiffness, ductility and toughness.

\section{Materials and methods}

\subsection{Materials}

The matrix material used in the fabrication of composite material was a mixture of Araldite epoxy resin (LY556) and hardener (HY951) mixed thoroughly in mass ratio (10:1). Three different fillers, namely rice husk, coconut shell powder and teak wood powder of two different sizes ranges from 75 - $105 \mu \mathrm{m}$ and $106-180 \mu \mathrm{m}$ were used as particulate reinforcements. The fillers were added in different mass percentages of matrix of 2.5 and 4.5 to obtain different levels of reinforcements.

\subsection{Processing of fillers}

The fillers were obtained by grinding the respective raw materials to fine powders of size ranging in microns using a grinding machine. The grinding process increased surface roughness and contact area of the fillers, which favoured mechanical interlocking with the matrix. Mesh 85, 150 and 200 were used to sieve the fillers according to BS 410-1:2000 [25] to separate them in two different size ranges $75-105 \mu \mathrm{m}$ and $106-180 \mu \mathrm{m}$. Distilled water was used to wash the fillers to remove any dirt, and filtered using clean white cloth. They was then dried in an oven at $120^{\circ} \mathrm{C}$ for 4 hours to remove the moisture. The fillers were inspected under a digital optical microscope to study surface roughness and shape. The optical microscopic images of various fillers, as shown in Fig. 1, provide information about the nature and shape of different fillers used. Tapped apparent density of the fillers was measured 
by pouring the filler in a container and tapped it 100 times in an ultrasonic agitator [26].

Table 1 provides detail information about the shape, density, cellulose and lignin contents of various fillers.

\subsection{Preparation of particulate filler composites}

Fillers that were ground to required size were heat treated in an oven at $120{ }^{\circ} \mathrm{C}$ for 4 hours to remove any moisture since bio fillers are extremely hydrophilic. The hardener HY951 was added into the Epoxy resin LY556 in mass ratio 10:1 and stirred well. Dried filler in different mass percentages of resin as required was mixed with the epoxy-hardener mixture. The mixture was stirred thoroughly for $8-10$ minutes to form a homogenous suspension. Composite material was producedby pouring the resin filler mixture into a mould and curing at room temperature for 24 hours. Details of different combinations of composite materials are presented in Table 2 .

\subsection{Indentation test}

Different types of composites were subjected to indentation load with reference to ASTM D 6264 for rigid support configuration [27], as shown in Fig. 2. Specimen size of length $\times$ width $\times$ thickness $(50 \times 50 \times 10 \mathrm{~mm})$ was used. Single and multi-cyclic loading were performed. Both displacement control and load control modes of testing were used for single loading. A loading rate of $2000 \mathrm{~N} / \mathrm{min}$ until it reached $8000 \mathrm{~N}$ was applied for the load control test. During displacement control test, a feed rate of $0.25 \mathrm{~mm} / \mathrm{min}$ was applied until $1.5 \mathrm{~mm}$ displacement. For multiple indentations, a cyclic load was applied in which a displacement increment of $0.75 \mathrm{~mm}$ was given for each cycle with a displacement feed rate of $0.25 \mathrm{~mm} / \mathrm{min}$.

\section{Results and discussion}

\subsection{Determination of deformability from indentation load control test}


A load of $8000 \mathrm{~N}$ was applied at a rate of $2000 \mathrm{~N} / \mathrm{min}$ and the deformation was measured for all specimens. This provides knowledge about the deformability of the materials. The load applied was the same for all specimens; consequently, the deformation was higher for ductile materials than the stiffer ones. The factors contributing to material stiffness are elastic property of the neat matrix itself, matrix filler interaction and degree of filler impregnation by resin and total volume content of filler in the composite material. Matrix/filler interaction was influenced by aspect ratio of filler, nature of filler surface and ability of impregnation of filler by resin. Rice husk (RH) is highly impregnable by resin so that it mixed thoroughly with resin, leaving no suspended particles, to form a homogeneous mix, unlike other fillers which were less easy for resin to impregnate. As the filler content increased, the viscosity of resin increased. This resulted in difficulty in impregnation and leaving some particles suspended in the matrix system. Even although Teak wood fillers are flat flakes, they are harder than RH which can be seen from the density values in Table 1. It was observed that more particles were suspended in the matrix, but they have rougher surface than the RH and coconut shell (CC) fillers, which aided high particle matrix interaction. Also, TW have more cellulose content, as given in Table 1, which have more hydroxyl groups that favour matrix particle interaction, as proposed in [9]. The composition of RH, CC and TW were taken from $[18,25]$. As for coconut shell with high lignin content, it is highly stiff and rigid. It is difficult to impregnate, but having rough surface and irregular 3D shape with pointed edges provides high shear and fractures resistance by deviating the crack. As far as the weight percentage of fillers is concerned, it is important that matrix wets the fillers so that there is enough filler matrix interaction in order to facilitate better load transfer between particles. The percentage volume content of fillers in various particulate composite laminates was determined from the respective filler densities and filler mass percentage from Tables 1 and 2. 
Fig. 3 shows the comparison of percentage volume content in each particulate composite laminate. Filler with high density like coconut shell occupied less volume, while low density filler like rice husk has high percentage of volume content in the particulate composite material for the same mass percentage of filler.

The deformation resulting from a load of $8000 \mathrm{~N}$ on various materials combinations is shown in Fig. 4. It is evident from the plots for the same load of $8000 \mathrm{~N}$, the deformations were not the same for different materials. Figs. 4(a)-(c) show that, for the same filler type and size, the deformability varies with filler mass percentage, which might be due to the fact that filler addition changed the nature of matrix to ductile. The mean distance between the particles was high for lower volume concentration which allowed the polymer chain to move, and hence resulted in high ductility. As the volume concentration became higher, the mean distance between the particles decreased and the polymer mobility was constrained. This caused the loss of ductility and increase in stiffness. From Figs. 4(g)-(i), it is evident that, even although the filler size and mass percentage remain same, there is variation in deformability due to the difference in type of filler. Therefore, it is evident that the filler properties like surface roughness, wettability of filler, shape, cellulose and lignin content play a major role in deformability. TW, which was moderately impregnable by resin, with high surface roughness and cellulose content possesses higher deformability, followed by RH which was highly impregnable by resin, but relatively soft, with less rough surface. This is followed by CC which was highly rigid. It is worth noting that filler size could also influence the deformability, as shown in Fig. 4(e) due to difference in matrix-filler interactions and availability of resin for impregnation. Fig. 4 shows that the stiffness of the materials keeps on varying throughout the course of loading. Initially, the stiffness was very low which led to high deformation, and then the stiffness increased, which might be due to the fact that the fillers in the subsequent layers beneath the surface accumulated to offer additional stiffness. 
Finally, when the material takes sufficient damage, the stiffness decreases again. The accumulation effect was evident, as depicted in Figs. 4(c) and in 4(f), where curves cross each other due to increase in stiffness during loading.

\subsection{Determination of stiffness from indentation displacement control test}

Figs. 5 (A)-(C) show the variation of stiffness with deformation interval of $0.25 \mathrm{~mm}$ in displacement control mode of testing and Figs. 5 (a)-(c) show the respective forces. It is seen that, for TW, the stiffness is continuously less than neat epoxy which indicates that it was very ductile, as discussed previously. In the case of $\mathrm{CC}$, they were relatively stiffer and some combinations even stiffer than neat epoxy due to their rigid nature. Stiffness variation during loading due to filler accumulation is evident from the curves crossing each other, and a similar effect was discussed by Rout and Satapathy [12] in their work relating to the investigation of rice-husk filled epoxy hybrid composites.

\subsection{Determination of elastic recovery from multiple indentation test}

Every material has an element of elastic property which is stored partially or fully in the material. To study the elastic recovery capability, multiple indentations were induced by cyclic loading. From zero position, the indenter was forced by $0.75 \mathrm{~mm}$ into the material and pulled back to zero position to complete the first cycle and then the indenter was forced by 1.5 $\mathrm{mm}$ into the material and pulled back to zero position to end the second cycle, and repeated accordingly. Elastic recovery for both cycles 1 and 2 were determined, as shown in Fig. 6. The loading curve of cycle 2 started from $0.75 \mathrm{~mm}$ if there was no elastic recovery. However, in this study, it started earlier because material recovered elastically to some extent but was not fully recovered. If recovered fully, the loading curves of both cycles coincides. The elastic recovery for both cycles were determined, as shown in Fig. 6 and Table 3.

\subsection{Damage acceptance and resistance of composite laminates}


When a load is applied to the material, strain energy is stored in the material. This strain energy is dissipated or restored based on the behavior of the material. If it causes any damage, the strain energy is absorbed or dissipated, otherwise the strain energy is restored once the load or strain is removed. A material with high ductility dissipates much strain energy by accepting damage, and a material with high elasticity restores much of the strain energy by resisting the external load. In load control tests, load is applied to the material and amount of damage incurred was measured from the strain energy absorbed. Material with high ductility accepted huge damage, as shown in Fig. 7 (C). In contrast, induced damage was given to the material in the displacement control test and the resistance offered by the material in the form of load was measured. Stiffer material offers better resistance to damage than the ductile one as determined by the higher area under the load-displacement curve obtained from displacement control test illustrated in Fig. 7 (b). Strain energy associated with both load and displacement control tests for different particulate composite material is given in Table 3.

The amount of total strain energy associated with each material is shown in Fig. 8, but the results of the percentage of strain energy absorbed and restored by each material is more relevant to the prediction of their properties. These were determined from the multi indentation test of the materials under cyclic loading. During cyclic loading, the indenter is removed in order to allow the material to recover and thereby allowing the estimation of strain energy restored. The amount of total strain energy inflicted on the material, amount of strain energy restored on the removal of indenter and amount of strain energy dissipated or absorbed due to the permanent damage incurred in the material was determined for cycle 1 , as shown in Fig. 8.

Fig. 8(a) shows the loading curve for cycle 1 and the area under the curve determines the strain energy induced on the material. The indenter was withdrawn allowing the material 
to recover and restore some energy. This is estimated from the loading curve for cycle 2 . It is observed that, if the material is recovered fully, the loading curve for cycle 2 will coincide with that of cycle1. However, in this study, as shown in Fig. 8(b), loading curve for cycle 2 traces another path which shows that it loses some energy. It was able to restore most of the energy mentioned as A2 in Fig. 8 but it failed to recover as much as energy mentioned as (A1 - A2) because of the permanent damage inflicted and the energy dissipated or absorbed in this process. In a similar way, the induced, restored and absorbed energy values were determined for cycle 2 .

A comparison of induced, restored and absorbed energy values of different particulate composite materials with different filler sizes and mass percentage combinations for two cycles is shown in Fig. 9. It is evident that filler type, filler size and filler mass percentage have influence over the energy absorption and restoration capabilities of the composite laminate. As expected, the restoration capability goes down from cycle1 to 2 due to the fact that in cycle 2 the material begins to accept the damage, and hence the energy absorption goes up. Comparatively, among the filler type used, the CC exhibits highest restoring capability, followed by TW and RH has the lowest.

The influence of size, shape, composition and volume content of various fillers on mechanical properties like elasticity, deformability, stiffness, and energy absorption capability have been discussed. No material is superior in all the above properties. Each material has its strength and weakness. Suitable material with respects to a certain property could be selected based on the requirement of the application. Therefore, the data for certain properties were normalized and plotted against each other, as shown in Fig. 10. This gives an overall idea of which combination is good or worse for a certain property. Based on this, it is possible to select the best material for a given application. 


\section{Conclusions}

From the investigations carried out on various combinations of filler type, size and mass percentage of particulate composites using single indentation load control, single indentation displacement control nd multi-cyclic indentation tests, the following conclusions can be deduced:

i. Filler size, mass percentage and filler type (chemical composition and shape) have influence on the deformability of the particulate composites. Among these, filler type is the most influential parameter. TW filler which is flat flake shaped with high cellulose content and surface roughness showed better deformability due to better matrix filler interaction and interlocking.

ii. CC particulate composite is stiffer than both TW and RH due to higher lignin content and spherical shaped particles. RH fillers which are rod and flake shaped with high cellulose content have higher tendency to be impregnated by resin making the material softer but showed high stiffness variation on loading. Thiswas based on their ability to deform and accumulate during the course of loading which provided additional stiffness.

iii. Availability of neat matrix, nature and ability of the filler to be impregnated by resin and total volume content of filler in the composite material are the factors that contribute to their elasticity. CC filler with lower impregnation ability due to high lignin content, has better elasticity in general. Also, increase in volume content reduces the elasticity and it becomes stiff and brittle. This is caused by the reduced mean distance between particles that restrict polymer sliding. Whereas in the case of fillers with high resin impregnation capability, such as RH and TW, elasticity increased with volume content provided there is enough matrix to wet and impregnate the fillers and bind the particles together. This effect might be due to the filler accumulation under compressive loading. 
iv. The strain energy absorption and restoration capability depended on the deformability and elastic recovery of the material. The CC filler particulate composite material has better strain energy restoration capability, while the RH has better strain energy absorption capability. Energy absorption capability was high for high volume content due to high matrix and particulate filler interactions helping energy dissipation effectively.

\section{References}

[1] F, Almansour, H.N. Dhakal, Z. Y. Zhang (2015). Effect of hybridisation on the mode II fracture toughness properties of flax/vinyl ester composites. Polymer Composites; 08/2015; DOI:10.1002/pc.23743.

[2] T.Lyashen Ko, N. Lerman, A. Wolf, H. Harel, G. Marom. Improved mode II delamination fracture toughness of composite materials by selective placement of protein-surface treated CNT, Composites Science and Technology 85 (2013) 29-35.

[3] J.M. Wernik, S.A. Meguid, On the mechanical characterization of carbon nanotube reinforced epoxy adhesives, Mater. Des. 59 (2014) 19-32.

[4] C. Okolieocha, F. Beckert, M. Herling, J. Breu, R. Mülhaupt,V. Altstat, Preparation of microcellular low-density PMMA nanocomposite foams: Influence of different fillers on the mechanical, rheological and cell morphological properties, Compos. Sci. Technol. 118 (2015) 108-116.

[5] A. M. Amaro, L Bernardo, DG. Pinto, SLopes, J Rodrigues, Cristina S. Louro, Effect of irregular shaped nanoalumina on the enhancement of mechanical properties of epoxy resin nanocomposites using DDM as hardener, Composites Part B 84 (2016) 17-24.

[6] D. Pinto, L. Bernardo, A. Amaro, S. Lopes, Mechanical properties of epoxy nanocomposites using titanium dioxide as reinforcement - A review, Construction and Building Materials 95 (2015) 506-524. 
[7] F.A. Alzarrug, M.M. Dimitrijević, R.M.J. Heinemann, V. Radojević, D.B. Stojanović, P.S. Uskoković, R. Aleksić, The use of different alumina fillers for improvement of the mechanical properties of hybrid PMMA composites, Mater. Des. 86 (2015) 575-581.

[8] L.C. Herrera-Ramírez, P. Castell, J.P. Fernández-Blázquez, Á. IFernández, R.G. de Villoria, How do graphite nanoplates affect the fracture toughness of polypropylene composites?, Compos. Sci. Technol. 111 (2015) 9-16.

[9] V. Fombuena, L. Bernardi, O.Fenollar, T. Boronat, R. Balart, Characterization of green composites from biobased epoxy matrices and bio-fillers derived from seashell wastes, Mater. Des. 57 (2014) 168-174.

[10] Z. Huang, N. Wang, Y Zhang, H. Hu, Y. Luo, Effect of mechanical activation pretreatment on the properties of sugarcane bagasse/poly(vinyl chloride) composites, Composites: Part A 43 (2012) 114-120.

[11] M. A. AlMaadeed, Z. Nogellova, M. Micusik, I. Novak, I. Krupa, Mechanical, sorption and adhesive properties of composites based on low density polyethylene filled with date palm wood powder, Mater. Des. 53 (2014) 29-37.

[12] A. K. Rout, A. Satapathy, Study on mechanical and tribo-performance of rice-husk filled glass-epoxy hybrid composites, Mater. Des. 41 (2012) 131-141.

[13] T. Shah, C. Gupta, R.L. Ferebee, M. R. Bockstaller, N. R. Washburn, Extraordinary toughening and strengthening effect in polymer nanocomposites using lignin-based fillers synthesized by ATRP, Polymer 72 (2015) 406-412.

[14] L. Averous, F. Le Digabel, Properties of biocomposites based on lignocellulosic fillers, Carbohydrate Polymers 66 (2006) 480-493.

[15] R. Liu, Y. Peng, J. Cao, Y. Chen, Comparison on properties of lignocellulosic flour/polymer composites by using wood, cellulose, and lignin flours as fillers, Compos. Sci. Technol. 103 (2014) 1-7. 
[16] I. Naghmouchi, F. X. Espinach, P. Mutjé, S. Boufi, Polypropylene composites based on lignocellulosic fillers: How the filler morphology affects the composite properties, Mater. Des. 65 (2015) 454-461.

[17] P. Leiva, E.M. Ciannamea, R.A. Ruseckaite, P.M. Stefani, Medium-density particleboards from rice husks and soybean protein concentrate, J. Appl. Polym. Sci. 106 (2007) 1301-1306.

[18] I. Miranda, V. Sousa, H. Pereira, Wood properties of teak (Tectonagrandis) from a mature unmanaged stand in East Timor, J Wood Sci (2011) DOI 10.1007/s10086-0101164-8.

[19] Z. Huang, N. Wang, Y. Zhang, H. Hu, Y. Luo, Effect of mechanical activation pretreatment on the properties of sugarcane bagasse/poly(vinyl chloride) composites, Composites: Part A 43 (2012) 114-120.

[20] M.A. AlMaadeed, Z. Nogellova, M. Micusik, I. Novak, I. Krupa, Mechanical, sorption and adhesive properties of composites based on low density polyethylene filled with date palm wood powder, Mater. Des. 53 (2014) 29-37.

[21] L. Averous, F.L. Digabel, Properties of biocomposites based on lignocellulosic fillers, Carbohydrate Polymers 66 (2006) 480-493.

[22] H.G.B. Premalal, H. Ismail, A. Baharin, Comparison of the mechanical properties of rice husk powder filled polypropylene composites with talc filled polypropylene composites, Polymer Testing 21 (2002) 833-839.

[23] H. S. Yang, M. P. Wolcott, H.S. Kim, S. Kim, H. J. Kim, Properties of lignocellulosic material filled polypropylene bio-composites made with different manufacturing processes, Polymer Testing 25 (2006) 668-676.

[24] BS 410-1: Test sieves - Technical requirements and testing - Part 1: Test sieves of metal wire cloth. British Standards Society (BSS) 2000. 
[25] ASTM B 527 - 93: Standard Test Method for Determination of Tap Density of Metallic Powders and Compounds. American Society for Testing and Materials (ASTM) 2000.

[26] ASTM D 6264 - 98: Standard Test Method for Measuring Damage Resistance of Fiber-Reinforced Polymer-Matrix Composite to Concentrated Quasi-Static Indentation Force. American Society for Testing and Materials (ASTM) 2004.

[27] Journal of the American Chemical Society (JACS) DOI: 10.1021/ja0127a068, 60(6), (1938) 1506-1511. 